\title{
Traumatic Incomplete Rupture of the Gastric Wall: Endoscopic Treatment With Clip Application
}

A 76-year-old woman, was involved in a high-speed car accident. The following diagnoses were made upon admission to our emergency unit: rupture of liver segment IV; liver hematoma (subcapsular) in segment VI; contusion of the pancreas; rupture of the spleen; rupture of the left accessory hepatic artery; and a Weber type $C$ fracture of the right ankle joint. A fracture of the nose with impression was also diagnosed.

The patient was immediately taken to the operating theatre where splenectomy, ligation of the left hepatic artery, and hemostasis of the liver capsule and the blood vessels in the gastrocolic ligament were performed. When the patient was transferred to the intensive care unit for further recovery, gastric bleeding was suspected because fresh blood was found in the gastric tube. At gastroscopy an incomplete rupture of the stomach wall with active bleeding was detected (Fig. 1a). Six metal clips were placed for hemostasis and closure of the mucosal defect (Fig. 1b).

At control endoscopy 1 week later, the mucosal rupture was completely closed and no further bleeding was evident. All six metal clips were still in place (Fig. 2). The patient recovered from general trauma and was discharged to an intermediate care ward 2 weeks after admission. The patient then progressed to show signs of further recovery. Surprisingly, 4 weeks after the initial discharge the patient died from an unknown cause; pulmonary embolism was discussed.

Despite the finally unfavorable outcome in our patient, we found endoscopic clip application to be a safe and reliable technique in the treatment of an incomplete gastric rupture in a critically ill patient,
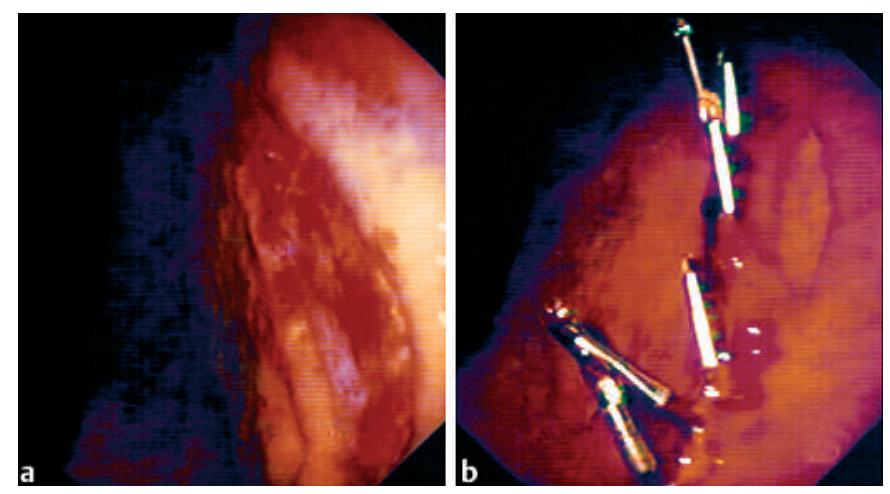

Figure 1 a The incomplete stomach wall rupture was inspected and showed stigmata of fresh bleeding, but no localized vessel or vascular stump was detected. b Six metal clips were placed to stop the bleeding and close the mucosal defect for further healing.

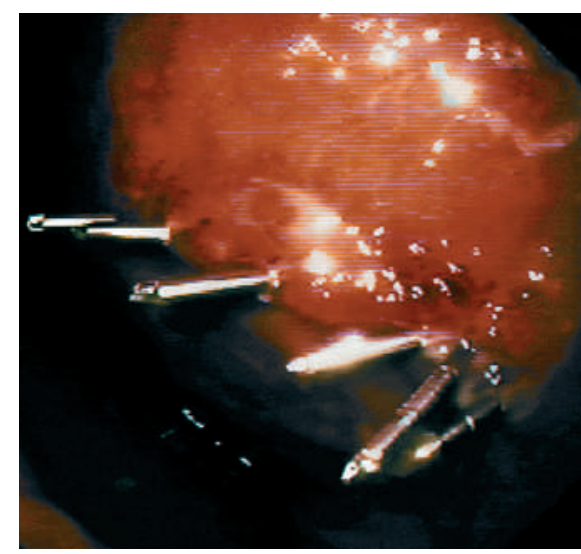

Corresponding Author

S. K. Gölder, M.D.

Department of Internal Medicine I University of Regensburg 93042 Regensburg Germany

Fax: $\quad$ +49-941-9447073

E-mail: stefan.goelder@klinik.uniregensburg.de
Figure 2 Control endoscopy of the gastric wall defect 1 week after clip placement. No further bleeding had occurred, and the six metal clips were still securely in place 먼 (Video 1).

as the endoscopic approach avoided any unnecessary overtreatment.

\section{S. Gölder ${ }^{1}$, M. Jeschke ${ }^{2}$, K. Jauch ${ }^{2}$,}

J. Schölmerich ${ }^{\mathbf{1}}, \mathbf{H}$. Messmann ${ }^{\mathbf{1}}$

${ }^{1}$ Department of Internal Medicine I, University of Regensburg, Regensburg, Germany

2 Department of Surgery, University of Regensburg, Regensburg, Germany.
Video 1 Traumatic incomplete gastric wall rupture: Endoscopic treatment with clip application. 